\title{
Numerical study of swirling flow in a cylinder with rotating top and bottom
}

\author{
Shen, Wen Zhong; Sørensen, Jens Nørkær; Michelsen, Jess
}

Published in:

Physics of Fluids

Link to article, DOI:

$10.1063 / 1.2204634$

Publication date:

2006

Document Version

Publisher's PDF, also known as Version of record

Link back to DTU Orbit

Citation (APA):

Shen, W. Z., Sørensen, J. N., \& Michelsen, J. (2006). Numerical study of swirling flow in a cylinder with rotating top and bottom. Physics of Fluids, 18(6), 064102. https://doi.org/10.1063/1.2204634

\section{General rights}

Copyright and moral rights for the publications made accessible in the public portal are retained by the authors and/or other copyright owners and it is a condition of accessing publications that users recognise and abide by the legal requirements associated with these rights.

- Users may download and print one copy of any publication from the public portal for the purpose of private study or research.

- You may not further distribute the material or use it for any profit-making activity or commercial gain

- You may freely distribute the URL identifying the publication in the public portal

If you believe that this document breaches copyright please contact us providing details, and we will remove access to the work immediately and investigate your claim 


\title{
Numerical study of swirling flow in a cylinder with rotating top and bottom
}

\author{
Wen Zhong Shen, ${ }^{\text {a) }}$ Jens Nørkær Sørensen, and Jess A. Michelsen \\ Department of Mechanical Engineering, Technical University of Denmark, DK-2800 Lyngby, Denmark
}

(Received 17 November 2005; accepted 11 April 2006; published online 6 June 2006)

\begin{abstract}
A numerical investigation of oscillatory instability is presented for axisymmetric swirling flow in a closed cylinder with rotating top and bottom. The critical Reynolds number and frequency of the oscillations are evaluated as function of the ratio of angular velocities of the bottom and the top $\left(\xi=\Omega_{\text {bottom }} / \Omega_{\text {top }}\right)$. Earlier linear stability analysis (LSA) using the Galerkin spectral method by Gelfgat et al. [Phys. Fluids, 8, 2614 (1996)] revealed that the curve of the critical Reynolds number behaves like an "S" around $\xi=0.54$ in the co-rotation branch and around $\xi=-0.63$ in the counter-rotation branch. Additional finite volume computations, however, did not show a clear $\mathrm{S}$ behavior. In order to check the existence of the $\mathrm{S}$ shape, computations are performed using an axisymmetric finite volume Navier-Stokes code at aspect ratios $(\lambda=H / R) 1.5$ and 2.0. Comparisons with LSA at $\lambda=1.5$ show that the S shape does exist. The $\mathrm{S}$ shape of the stability diagram predicted by LSA is thus confirmed by a finite-volume based Navier-Stokes solver. The additional computations at aspect ratio $\lambda=2$ show that the curve of critical Reynolds number has a wider $\mathrm{S}$ shape in the co-rotating branch for $\xi$ about 0.7 whereas a sharp "beak" appears in the counter-rotating branch for $\xi$ approximately -0.5. (C) 2006 American Institute of Physics.
\end{abstract}

[DOI: $10.1063 / 1.2204634]$

\section{INTRODUCTION}

The flow in a closed cylindrical container with a rotating lid has been studied both experimentally and computationally for more than three decades. The first experiments by Vogel $^{1}$ and later by Ronnenberg ${ }^{2}$ and Escudier ${ }^{3}$ showed the formation of a concentrated vortex core along the center axis. Based on their pioneering works, a lot of experimental and numerical studies have later been carried out. Among those, Spohn et al. ${ }^{4}$ used the visualization techniques of electrolytic precipitation and fluorescent dye and showed that vortexbreakdown bubbles in the container flow are open and asymmetric at their downstream end in the regime of axisymmetric bubbles. Recently, Sotiropoulos et al..$^{5}$ used planar laserinduced fluorescence technique and showed the existence of chaotic behavior in the Lagrangian transport within the bubbles for flows that are steady from the Eulerian standpoint. In computations, there are numerous investigations of the container problem, for example, Lopez, ${ }^{6}$ Brown and Lopez, ${ }^{7}$ Daube, ${ }^{8}$ Lopez and Perry, ${ }^{9}$ Sørensen and Christensen, ${ }^{10}$ Watson and Neitzel, ${ }^{11}$ Gelfgat et al. ${ }^{12}$ Tsitverblit and Kit, ${ }^{13}$ Sotiropoulos and Ventikos, ${ }^{14}$ Sotiropoulos et al.,${ }^{15}$ Blackburn and Lopez, ${ }^{16}$ Serre and Bontoux, ${ }^{17}$ and Blackburn. ${ }^{18}$ All these studies were able to capture the flow evolution observed in the laboratory.

Apart from one side rotation, rotating simultaneously the other end wall can give the problem more new insights and parametrically studying stability, bifurcations and flow structures can give some ideas on how to control vortex breakdown.

\footnotetext{
a) Author to whom correspondence should be addressed. Fax: (45) 459306 63. Electronic mail: shen@mek.dtu.dk
}

The influence of co- and counter-rotation of the other end wall of the cylinder on vortex breakdown was studied experimentally by Bar-Yoseph et al., ${ }^{19}$ Gautier et al.,${ }^{20}$ and Fujimura et $a l^{21}$ In computations, Valentine and Jahnke ${ }^{22}$ and Lopez ${ }^{23}$ studied the case of co-rotating end walls with the same angular velocity for steady and unsteady swirl flow. Nore et $a l^{24}$ studied the case of counter-rotating end walls for steady and unsteady swirl flow and determined that onset of three dimensionality for exactly counter-rotating end walls takes place at a Reynolds numbers of approximately 350 .

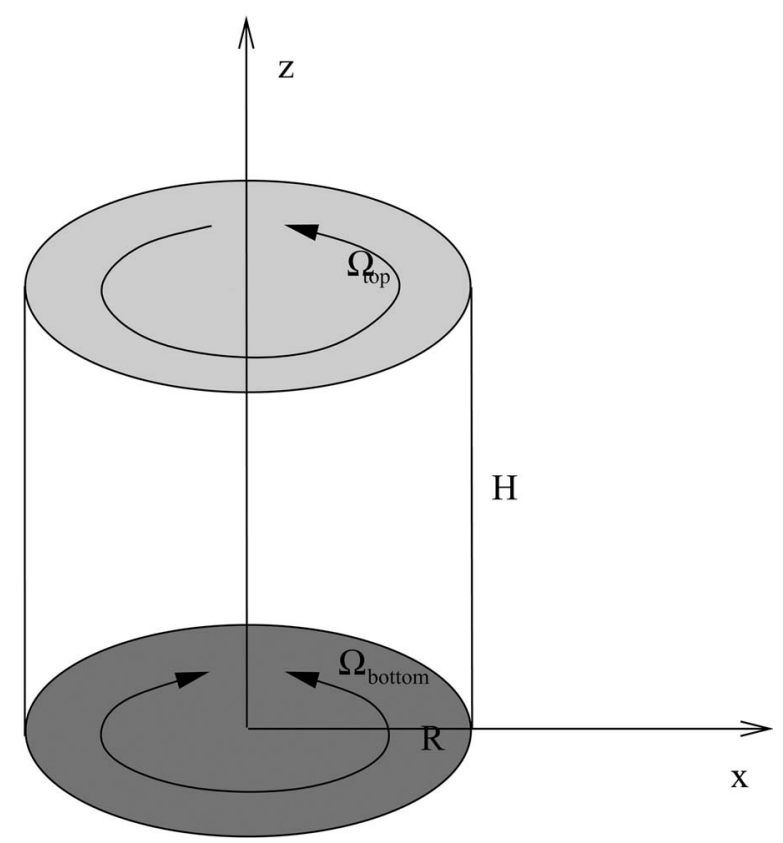

FIG. 1. Schematic representation of the flow geometry. 
(a)

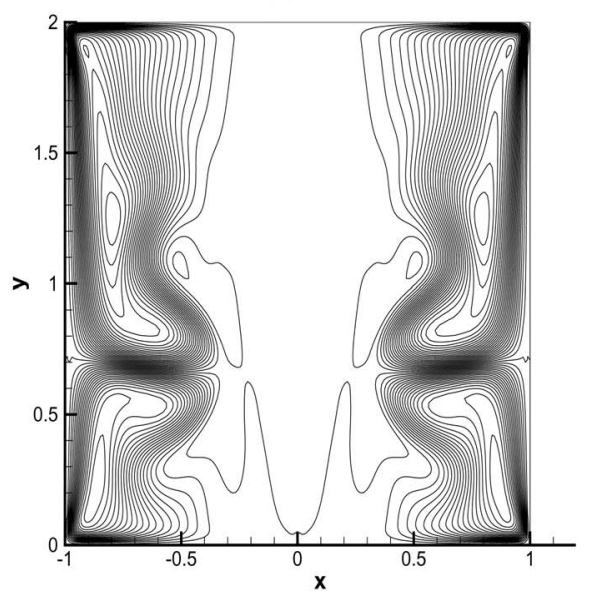

(b)

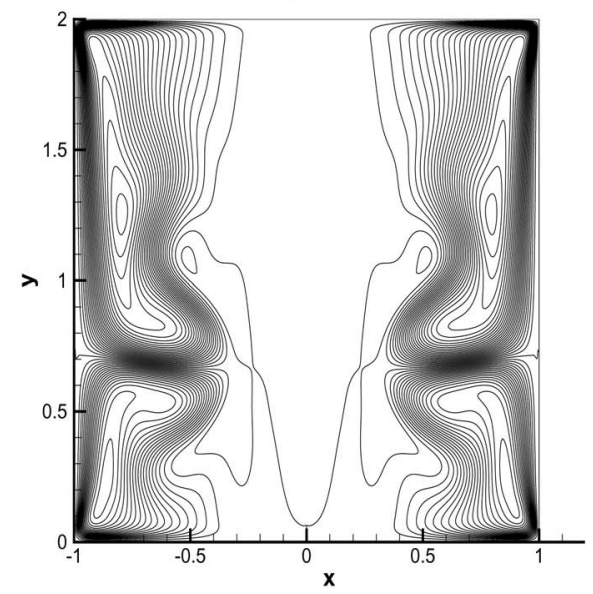

FIG. 2. Streamline plot of swirling flow in a cylinder with co-rotating top and bottom at $\lambda=2, \xi=0.7$, and $\mathrm{Re}$ $=4000$ on (a) a medium mesh consisting of $96 \times 128$ cells and (b) a fine mesh consisting of $192 \times 256$ cells.
Gelfgat et al. ${ }^{25}$ performed a parametric investigation of the oscillatory instability for the axisymmetric flow case at an aspect ratio of 1.5 . From their linear stability analysis, they found, at a ratio of angular velocities $\xi$ about 0.54 in the co-rotation branch, the existence of three distinct critical points with different critical frequencies. The curve of the critical Reynolds number around $\xi=0.54$ looks like an "S." In the counter-rotation branch, the same phenomenon was observed at a ratio of angular velocity $\xi$ approximately -0.63 . In the same article, finite volume computations were also performed. From the computations, using three different meshes, no such $\mathrm{S}$ shape of bifurcation could be seen. What happens here? Is it the linear stability method or the finite volume method that fails to predict the physics?

The focus of the present article is to investigate numerically the previous phenomenon using a code based on an axisymmetric finite volume method. We here present comparative results for the studied case of $\lambda=1.5$ as well as new results for $\lambda=2.0$. This article is organized as follows. In Sec. II, the numerical method is described. The validation of the code is shown in Sec. III. Numerical results and discussions are presented in Secs. IV-VI.

\section{FORMULATION OF THE PROBLEM AND NUMERICAL METHOD}

Consider a circular cylinder of radius $R$ and height $H$ filled with an incompressible Newtonian fluid of constant density $\rho$ and kinematic viscosity $\nu$. The top and the bottom of the cylinder are rotating with angular velocities $\Omega_{\text {top }}$ and $\Omega_{\text {bottom }}$, respectively (see Fig. 1). The various flow regimes are determined by the Reynolds number, $\operatorname{Re}=\Omega_{\text {top }} R^{2} / \nu$, the aspect ratio, $\lambda=H / R$, and the ratio of angular velocities, $\xi$ $=\Omega_{\text {bottom }} / \Omega_{\text {top }}$.

The flow is governed by the unsteady incompressible Navier-Stokes equations. Because of the simple geometry of the cylinder, it is natural to formulate the equations in cylindrical coordinates. The resulting Navier-Stokes equations are solved by a predictor-corrector method based on a cellcentered finite-volume/multiblock strategy. ${ }^{26,27}$ In the predictor step, the momentum equations are discretized using a second-order backward differentiation scheme in time and second-order central differences in space, except for the convective terms that are discretized by the QUICK upwind scheme. As all variables are defined at cell centers, no special treatment is needed for the singularity problem at the center axis. In the corrector step, the new Rhie-Chow interpolation developed by Shen et $a l^{28}$ and the new SIMPLE-C scheme on collocated grids ${ }^{29}$ are used in order to avoid numerical oscillations from pressure decoupling. The obtained pressure Poisson equation is solved by a five-level multigrid technique. For more details about the numerical technique, the reader is referred to Refs. 26-29. (a)

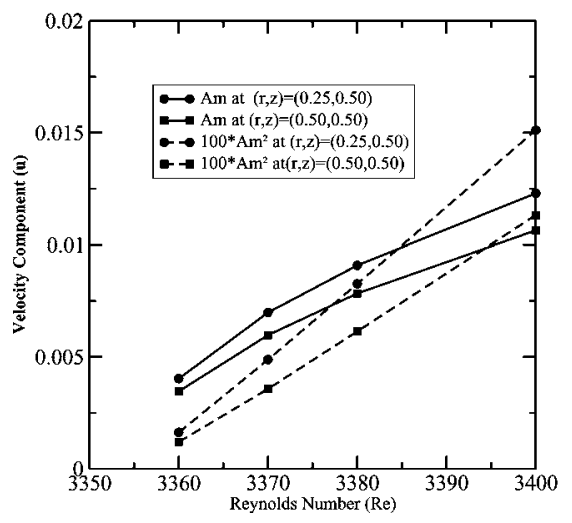

(b)

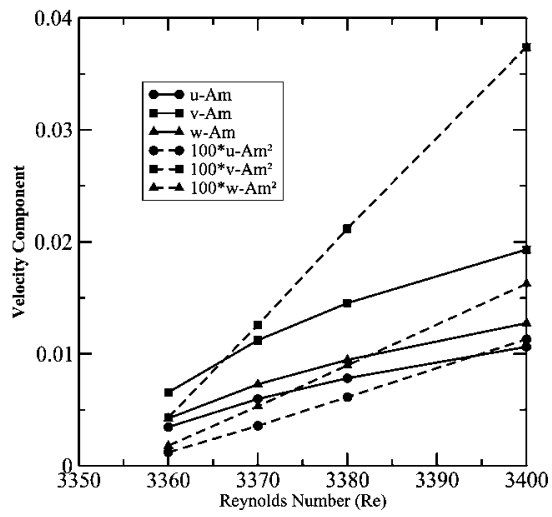

FIG. 3. Plot of velocity amplitudes and square amplitudes for swirl flow in a closed cylinder with counterrotating top and bottom $(\xi=-0.5)$. (a) Same velocity component at two different positions and (b) three velocity components. 
(a)

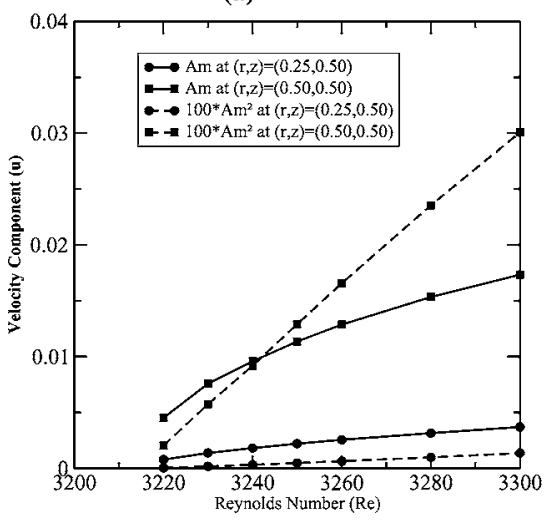

(b)

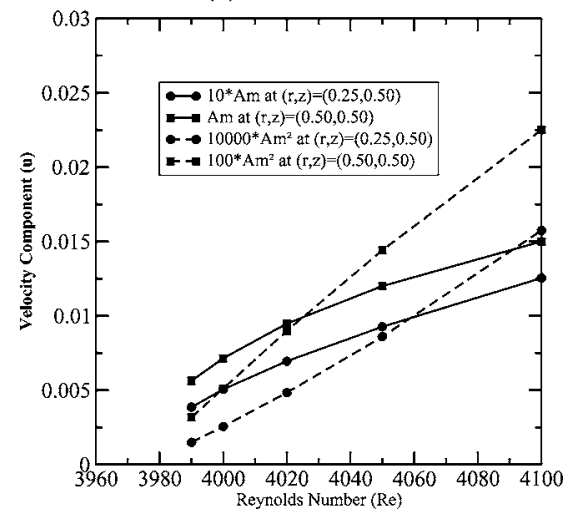

FIG. 4. Plot of velocity amplitudes and square velocity amplitudes for corotating top and bottom. (a) $\xi=0.5$ and (b) $\xi=0.8$.

\section{VALIDATION OF THE NUMERICAL CODE}

To validate the code, swirling flows in a cylinder with co- and counter-rotating end walls are computed and analyzed at an aspect ratio of $\lambda=2$. As parameter studies at various ratios of angular velocities, $\xi$, and Reynolds numbers are needed, a standard mesh of medium size is used in most of the computations. Based on experience gained from previous numerical studies ${ }^{10}$ a standard mesh (s) is defined that consists of 96 cells in radial direction and 128 cells in axial direction with stretching toward the walls and the center axis. In order to demonstrate mesh independence, a finer mesh (f) in which the number of cells is doubled in each direction, i.e., $192 \times 256$ cells, is also used. To evaluate the influence of the grid, the code is validated for the case of co-rotation at a ratio of angular velocities $\xi=0.7$ and a Reynolds number of 4000.

After a long transient, a steady state is reached. In Fig. 2 streamlines are plotted on the standard mesh and on the fine mesh. From the figure, only small differences can be seen. Defining the difference of maximum and minimum values of the stream function $\psi$ as $D=\psi_{\max }-\psi_{\min }$ and the relative error

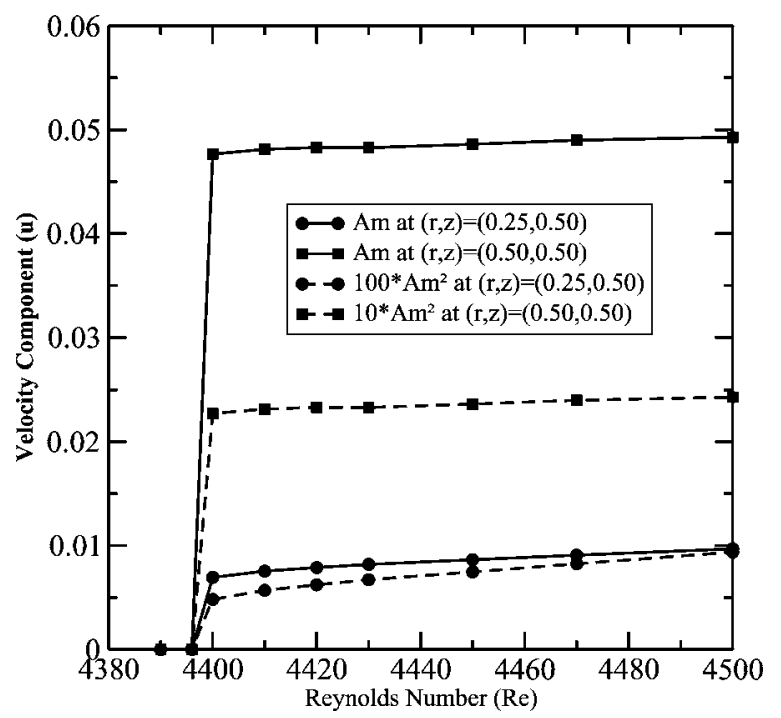

FIG. 5. Plot of velocity amplitudes and square velocity amplitudes for corotating top and bottom at $\xi=0.7$. between two meshes as $E_{r}=\left(D_{s}-D_{f}\right) / D_{f}$, the relative error is found to be $0.896 \%$. Therefore the standard mesh of medium size is deemed adequate for the present investigations.

\section{DETERMINATION OF CRITICAL REYNOLDS NUMBER}

Assuming that the transition from a steady to an unsteady regime sets in as a supercritical Hopf bifurcation, the critical Reynolds number can be determined from the amplitude of the velocity components that decreases linearly with the Reynolds number in the unsteady regime. Thus, the critical Reynolds number can be determined from

$$
\operatorname{Re}_{\mathrm{cr}}=\operatorname{Re}_{1}-\frac{A_{1}^{2}}{A_{2}^{2}-A_{1}^{2}}\left(\operatorname{Re}_{2}-\operatorname{Re}_{1}\right),
$$

where subscripts 1 and 2 refer to two different points in the unsteady region that are located close to the critical Reynolds number. In Fig. 3 the development is shown of the amplitude and the square of the amplitude of velocity components close

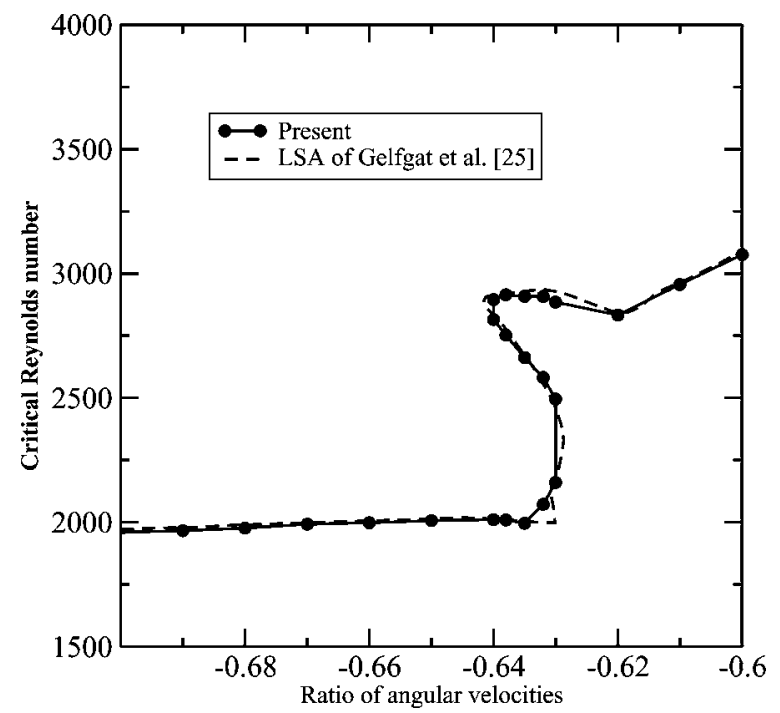

FIG. 6. Stability diagram of oscillatory instability in a cylinder of aspect ratio of 1.5 with rotating top and counter-rotating bottom. 


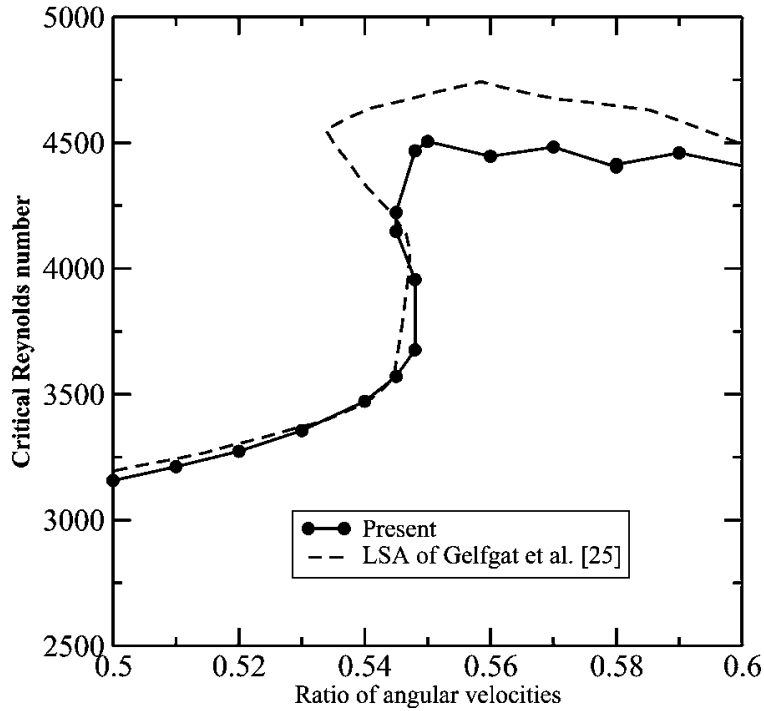

FIG. 7. Stability diagram of oscillatory instability in a cylinder of aspect ratio of 1.5 with rotating top and co-rotating bottom.

to the critical point at $\lambda=2$ and $\xi=-0.5$. The left-hand panel of Fig. 3 shows the distribution of amplitudes and square amplitudes for the $u$ component at two different positions, $(r, z)=(0.25,0.5)$ and $(r, z)=(0.5,0.5)$, and the right-hand panel of Fig. 3 shows the distribution of amplitudes and square amplitudes for all three velocity components at $(r, z)=(0.5,0.5)$. From Fig. 3 , it is seen that the square of the velocity amplitude decreases almost linearly with the Reynolds number (Re). Within numerical accuracy, the different curves predict almost the same critical Reynolds number.

In the co-rotating branch, the first bifurcation from a steady to an unsteady flow regime is also governed by a supercritical Hopf bifurcation. This Hopf bifurcation stops at the left-top end of the $\mathrm{S}(\xi \leqslant 0.663)$ and appears again after the S shape $(\xi \geqslant 0.74)$ (see also Fig. 13). Figure 4 shows the development of the amplitude and the square of the amplitude of velocity components close to the critical point. From Fig. 4, we can see that the square amplitude decreases monotonously with the Reynolds number. In the top region of the $\mathrm{S}$ shape $(0.663 \leqslant \xi \leqslant 0.73)$ the bifurcation, however, appears to differ from a supercritical Hopf bifurcation. The velocity amplitude and the square velocity amplitude are shown in Fig. 5. From Fig. 5, it is seen that both the velocity amplitude and the square velocity amplitude suddenly drops and exhibits a discontinuous behavior at the critical Reynolds number. Although we do not study the phenomenon further, it is most likely a subcritical Hopf bifurcation that sets in. In order to determine the critical Reynolds number for this bifurcation, a lot of computations at Reynolds numbers close to the critical Reynolds number were carried out. (a)

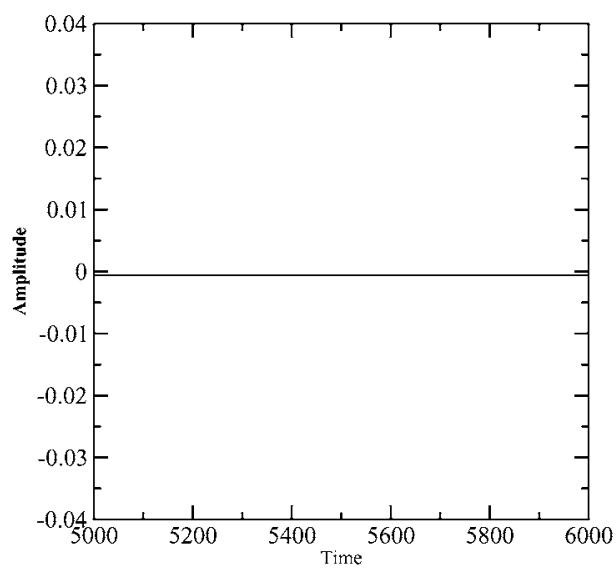

(c)

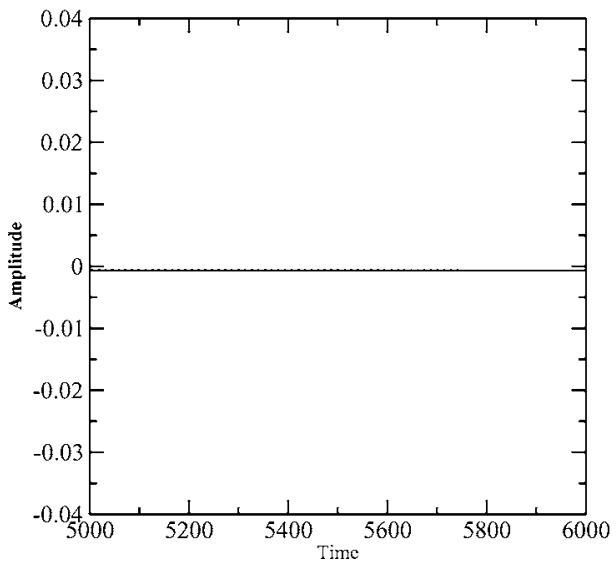

(b)

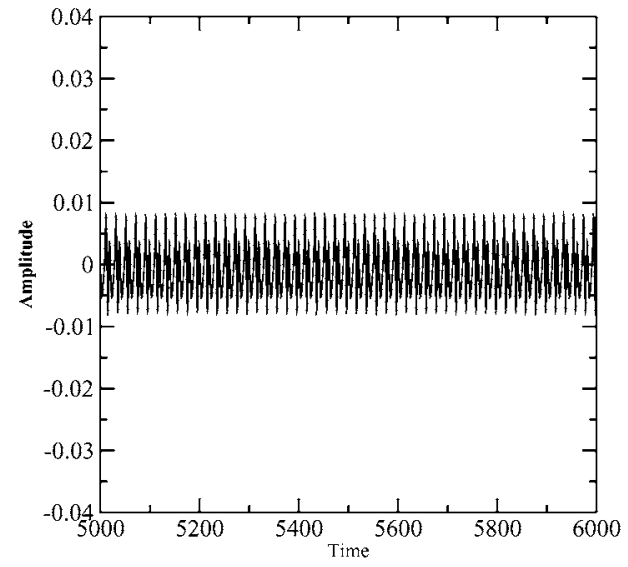

(d)

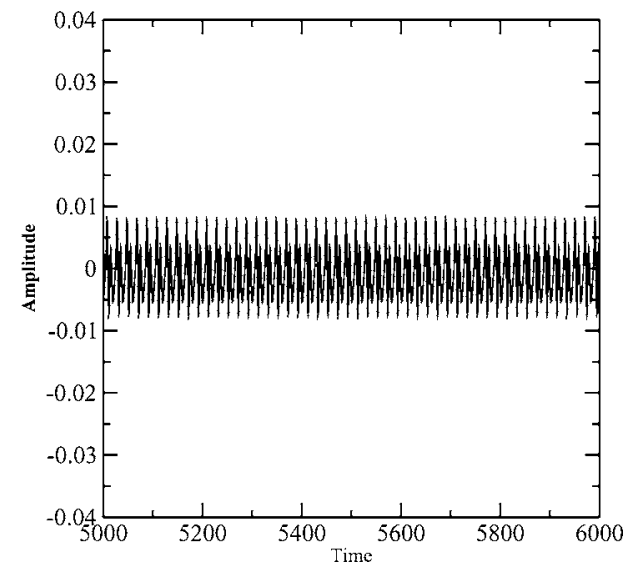

FIG. 8. Velocity signals at $(r, z)$ $=(0.25,0.375)$ for swirl flow in a closed cylinder with an aspect ratio 1.5 and co-rotating end walls of $\xi$ $=0.58$. (a) $\operatorname{Re}=4403$; (b) $\operatorname{Re}=4405$; (c) $\mathrm{Re}=4410$; and (d) $\mathrm{Re}=4415$. 
(a)

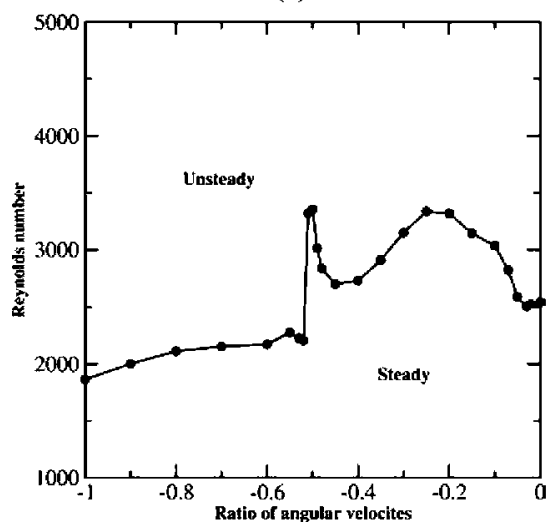

(b)

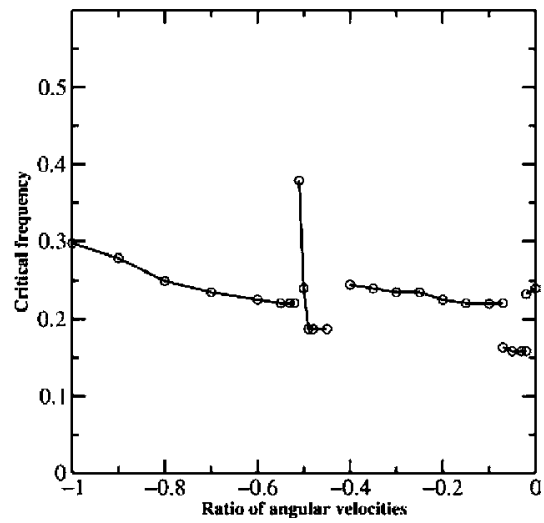

FIG. 9. Stability diagrams of oscillatory instability in a cylinder of aspect ratio 2 with rotating top and counterrotating bottom, (a) critical Reynolds number and (b) critical frequency.

\section{COMPARISON WITH LINEAR STABILITY ANALYSIS}

In order to check the existence of the $\mathrm{S}$ shape, computations are first performed at an aspect ratio $\lambda=1.5$, corresponding to the case considered by Gelfgat $e t a{ }^{25}$ Our attention, however, is mainly focused on the ratio of angular velocities $\xi$ in the regions where the $\mathrm{S}$ shape appears. Figure 6 shows stability diagrams of the critical Reynolds number in the counter-rotating region computed by the present finite volume code and the linear stability analysis (LSA) of Gelfgat et al. ${ }^{25}$ From Fig. 6, excellent agreement between the present method and linear stability analysis of Gelfgat is found and the $\mathrm{S}$ shape is very well captured by our finite volume method.

Figure 7 shows stability diagrams of the critical Reynolds number in the co-rotating region. From Fig. 7, the $\mathrm{S}$ shape is seen to be captured by our finite volume code, but the width of the S shape is smaller than that of LSA. Before reaching the $S$, the two stability curves are in good agreement. After the S shape, the critical Reynolds number computed by the present code is smaller than that predicted by LSA. One interesting point found during the computations is that a stability spot is found at $\xi=0.58$. The flow changes state from steady to unsteady when the Reynolds number reaches 4404 . The flow again becomes steady when the (a)

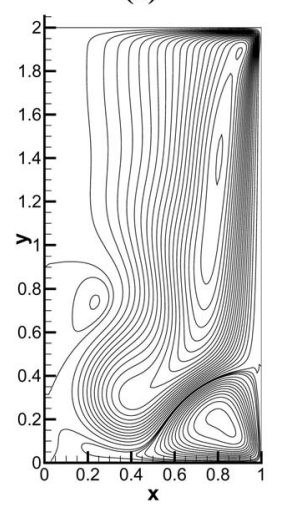

(b)

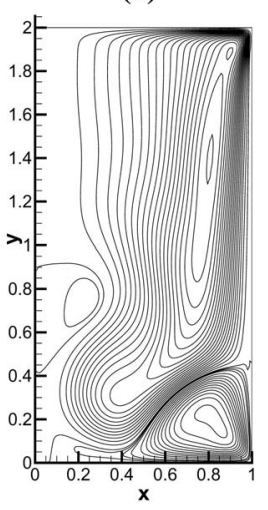

(c)

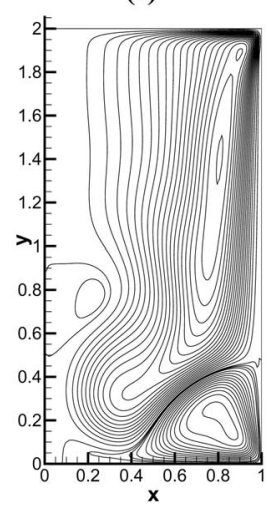

FIG. 10. Iso-lines of stream function. Counter-rotation at $\mathrm{Re}=3000$ and $-0.49 \geqslant \xi \geqslant-0.51$. (a) $\xi=-0.49$; (b) $\xi=-0.50$; and (c) $\xi=-0.51$.
Reynolds number is increased to 4408 , and stays steady until the Reynolds number exceeds 4413.5. After this point, the flow becomes unsteady. This phenomenon can be seen from velocity fluctuations at different positions (see Fig. 8).

\section{SWIRL FLOW AT ASPECT RATIO 2}

To supplement the computations at $\lambda=1.5$, computations were performed at an aspect ratio $\lambda=2$. First the influence of counter-rotation on the transition from steady to unsteady flow is studied. The dependence of the critical Reynolds number, $\operatorname{Re}_{\mathrm{cr}}$, on the ratio of angular velocities, $\xi$, is plotted in Fig. 9(a). From Fig. 9(a), we can see that when increasing slightly the counter-rotating angular velocity, i.e., by letting $\xi$ vary from zero to a small negative value, the critical Reynolds number decreases slightly. If counter-rotation increases more, the critical Reynolds number starts to increase rapidly, implying that the steady region for a moderate counterrotation has been enlarged. Further increasing counterrotation, the size of the steady region reaches a maximum and then decreases, forming a sharp "beak" around $\xi=-0.5$. The critical Reynolds number stays flat after the beak. The reduced frequency is defined as $\omega=2 \pi f / \Omega_{\text {top }}$, where $f(\mathrm{~Hz})$ is the frequency of oscillation. The critical reduced frequency, $\omega_{\text {cr }}$, as a function of the ratio of angular velocities is also plotted in Fig. 9(b). From Fig. 9(b), one can see that the (a)

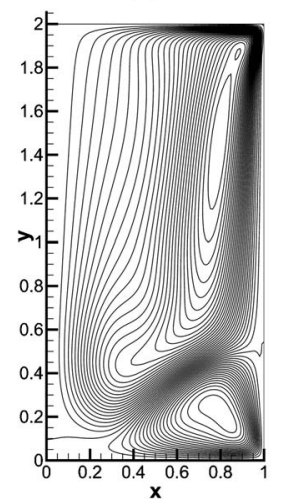

(b)

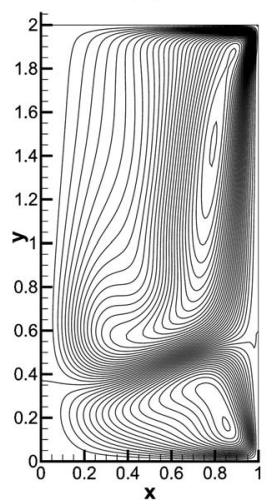

(c)

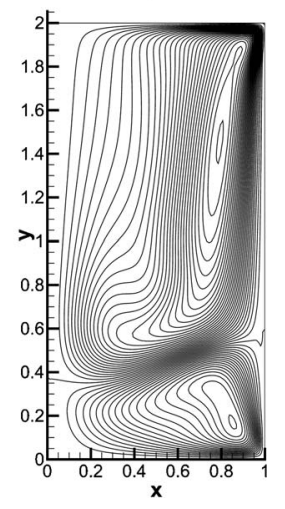

FIG. 11. Iso-lines of stream function. Counter-rotation at $\mathrm{Re}=2200$ and $-0.51 \geqslant \xi \geqslant-0.53$. (a) $\xi=-0.51$; (b) $\xi=-0.52$; and (c) $\xi=-0.53$. 
(a)

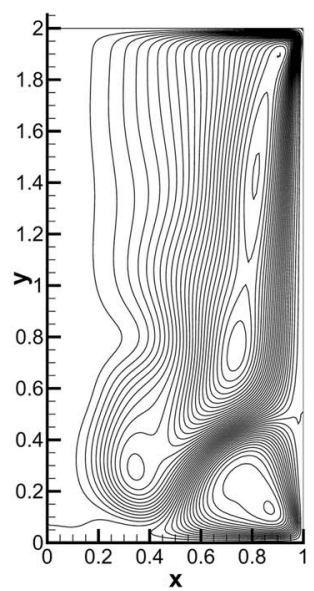

(b)

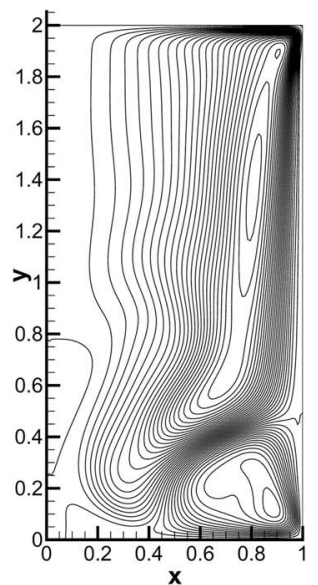

(c)

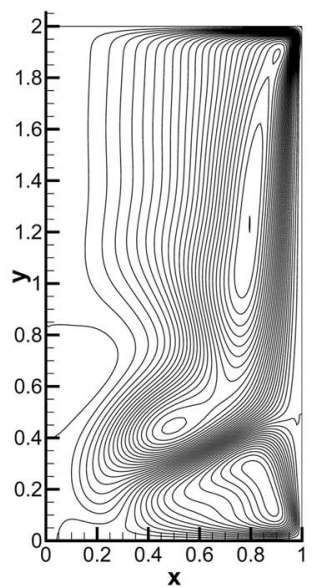

(d)

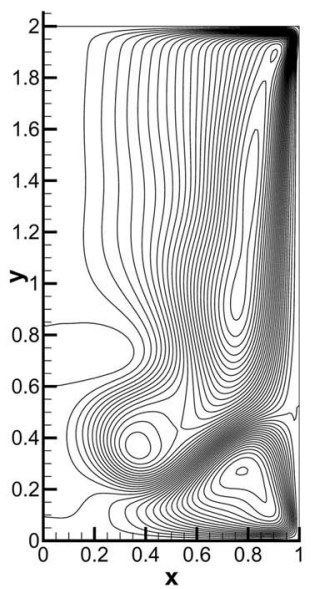

FIG. 12. Stream line plots for unsteady swirl flow in a closed cylinder with counter-rotating bottom at Re $=3250$, and $\xi=-0.52$. Four different time instants in a period (a) $t=t_{0}$; (b) $t=t_{0}+T / 4$; (c) $t=t_{0}+T / 2$; and (d) $t=t_{0}+3 T / 4$.

frequency of oscillation changes at $\xi=-0.02$ and $\xi-0.07$ from 0.23 to 0.16 and back from 0.16 to 0.22 , respectively, signalling the appearance of a different disturbance mode in this range. Similar changes of the dominant mode were revealed by Gelfgat $e t a l .^{25}$ Note that at $\xi=-0.02$, the dominant reduced frequency is 0.23 , but that the signal also contains a lower frequency component of 0.16 . At $\xi=-0.07$, the same phenomenon is observed. The reduced frequency follows another branch in the region close to the beak of the critical Reynolds number $(-0.51 \leqslant \xi<-0.4)$. If the counter-rotating angular velocity is further increased, the frequency increases slightly and returns to the original branch.

In order to clarify the behavior of the bifurcation around the beak of critical Reynolds number at $\xi=-0.51$, it is instructive to study the resulting flow structures. First, at a Reynolds number of 3000 , the parameter $\xi$ is varied from -0.49 to -0.51 . The corresponding streamlines are plotted in Fig. 10. From the picture, one can see that the intensity of the middle bubble as well as the size of the separation zone along the bottom edge changes slightly. Second, at a Reynolds number of 2200, the ratio of angular velocities of counter-rotation is varied from -0.51 to -0.53 . From Fig. 11, we can see that there is no middle bubble and that the bottom edge separation zone is bigger than the one in Fig. 10. From a topological point of view, two flow patterns are essentially different. The difference can also be found from the stability curve of critical frequency in Fig. 6 and they do simply not belong to the same branch. The unsteady flow structure is shown in Fig. 12. From the picture, we can see that the bottom edge separation size changes during one period and the middle bubble close to the center axis appears and then disappears. This situation can be considered as a mixed picture of the situations in Figs. 10 and 11. If the counterrotating velocity is increased further, the bottom bubble increases smoothly to end up as a symmetrical pattern at $\xi=-1$.

In the following, the influence of co-rotating on the transition from steady to unsteady flow will be studied. The dependence of the critical Reynolds number, $\mathrm{Re}_{\mathrm{cr}}$, on the ratio of angular velocities, $\xi$, is plotted in Fig. 13(a). From Fig. 13(a), we can see that increasing the co-rotating angular velocity increases the steady Reynolds number region. At angular velocities about $\xi=0.7$, an $\mathrm{S}$ shaped diagram is found. This S shape of critical Reynolds numbers was first found by Gelfgat et al. ${ }^{25}$ using linear stability theory in the case of a swirl flow in a closed cylinder with an aspect ratio of 1.5. Unfortunately, the structure could not be reproduced by their finite volume computations. The phenomenon at $\xi=0.7$ is (a)

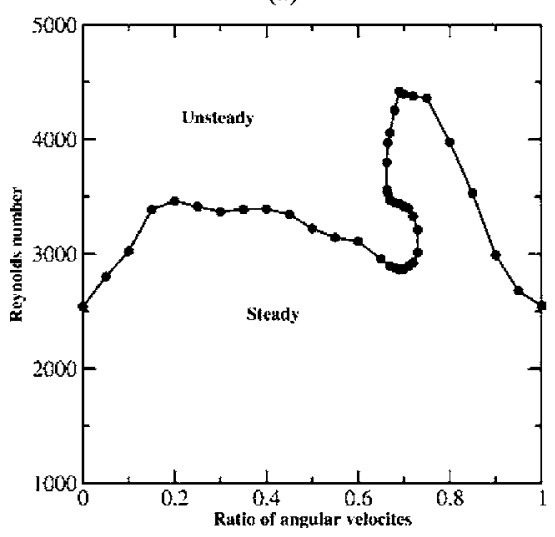

(b)

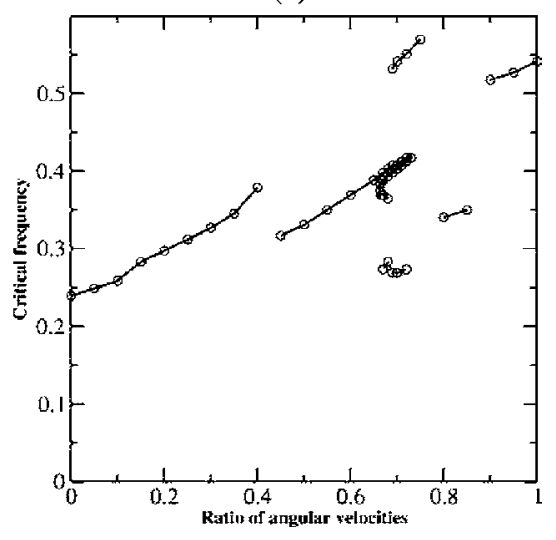

FIG. 13. Stability diagrams of oscillatory instability in a cylinder of aspect ratio 2 with rotating top and corotating bottom, (a) critical Reynolds number and (b) critical frequency. 
(a)

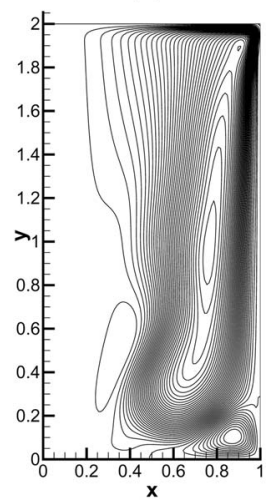

(b)

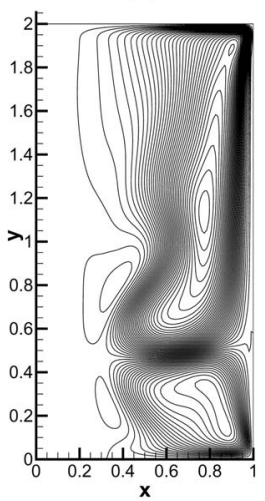

(c)

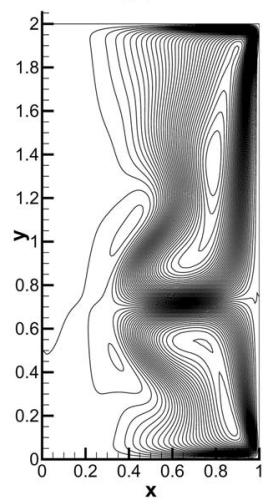

FIG. 14. Streamline plots of swirl flow in a closed cylinder with co-rotating bottom at: (a) $\operatorname{Re}=3300$ and $\xi=0.3$; (b) $\operatorname{Re}=3250$ and $\xi=0.5$; and (c) $\operatorname{Re}=2800, \xi=0.7$.

that the flow first becomes unsteady at a Reynolds number above 2870 and stays unsteady for Reynolds numbers up to 3418. The flow then becomes steady when the Reynolds number is further increased in the range from 3418 to 4398. The flow is fully unsteady at Reynolds numbers above 4398 .

The critical frequency in Fig. 13(b) increases continuously to $\omega_{\text {cr }}=0.379$ for ratios of angular velocities up to 0.4 and thereafter it is shifted to another branch $\left(\omega_{\mathrm{cr}}=0.316\right)$. It then stays on the same branch as long as the ratio $\xi$ remains in the first half (lower side) of the S. The frequency changes at $\xi=0.67$ in the second half (upper side) of the $\mathrm{S}$ into a lower frequency $\left(\omega_{\mathrm{cr}}=0.273\right)$. Shortly after $\xi=0.69$, a double harmonics appears in the signal. At $\xi=0.75$, this double harmonic frequency becomes the basic frequency. Thus, the lower frequency created at $\xi=0.67$ disappears at $\xi=0.75$. After that, another branch appears for $\xi \geqslant 0.8$ with a slightly lower frequency $\left(\omega_{\mathrm{cr}}=0.34\right)$. At $\xi=0.9$, the critical frequency appears on a branch with a high value $\left(\omega_{\mathrm{cr}}=0.518\right)$, where it stays continuously until $\xi=1\left(\omega_{\mathrm{cr}}=0.542\right)$.

To clarify in detail the flow behavior around the bifurcations it is instructive to look at the development of the flow structures. When increasing slightly the co-rotating velocity,

the size of the middle bubble close to the center axis increases for finally to detach from the center axis. At the same time, a small separation zone appears along the edge of the bottom wall. If the co-rotating angular velocity is further increased up to $\xi=0.7$, we see from Fig. 14 that the lower separation zone increases and pushes the upper bubble back toward the top wall. At $\xi=0.7$, the flow becomes unsteady at a Reynolds number of 2900. In Fig. 15, the stream function is plotted for one period. From Fig. 15, it is seen that the unsteadiness of the swirl flow is mainly caused by the structure in the lower side of the cylinder. The flow again stays steady for Reynolds numbers of 3500, 3800, 4000, and 4300 (see Fig. 16). From Fig. 16, we see that the flow structure is nearly identical at these Reynolds numbers. The unsteady flow pattern at a Reynolds number of 4400 is shown in Fig. 17. From Fig. 17, the flow is seen to be dominated by largescale fluctuating structures from both the top and the bottom of the cylinder.

For $\xi \geqslant 0.8$, all flow patterns have a similar structure. The lower side bubble increases in size when the co-rotating velocity increases and the flow becomes symmetrical at $\xi=1$.

\section{CONCLUSIONS}

The stability of steady flow and the onset of the oscillatory instability were studied by using a finite volume code for a closed cylinder with rotating top and bottom. From our computations at the same aspect ratio $\lambda=1.5$ as the earlier investigations by Gelfgat $e t$ al., the $\mathrm{S}$ shape of the stability curve is confirmed in agreement with the linear stability analysis. At an aspect ratio $\lambda=2$, our results show that the critical Reynolds number curve has a beak shape in the counter-rotation region and a much wider $\mathrm{S}$ shape in the co-rotation region. From linear stability theory for a cylinder at $\lambda=1.5$ the $\mathrm{S}$ shape did exist in both co-rotation and counter-rotation branches of the stability curve of the critical (a)

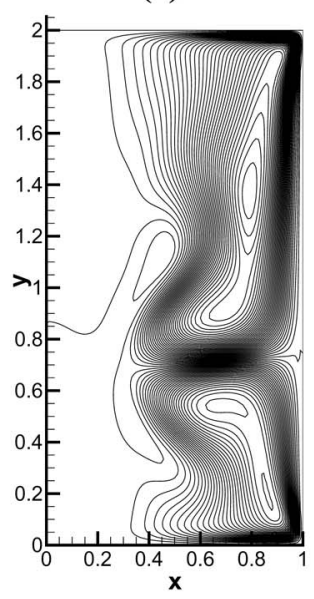

(b)

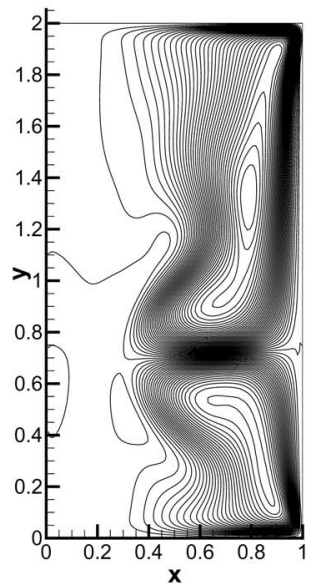

(c)

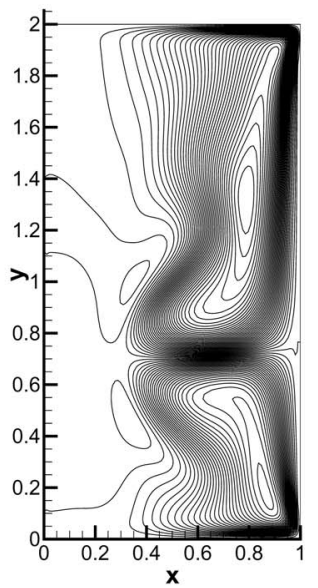

(d)

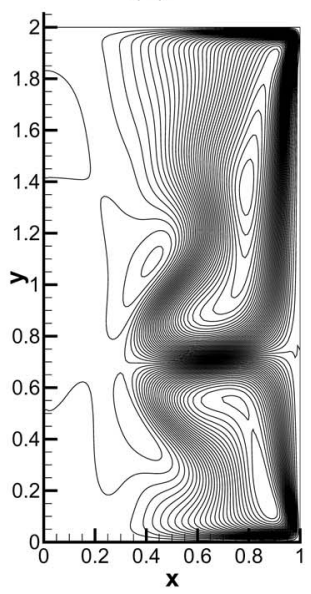

FIG. 15. Stream line plots for unsteady swirl flow in a closed cylinder with co-rotating bottom at $\operatorname{Re}=2900, \xi=0.7$. (a) $t=t_{0}$; (b) $t=t_{0}+T / 4$; (c) $t=t_{0}+T / 2$; and (d) $t=t_{0}+3 T / 4$. 
(a)

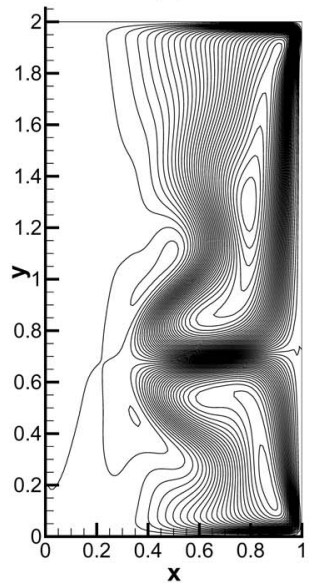

(b)

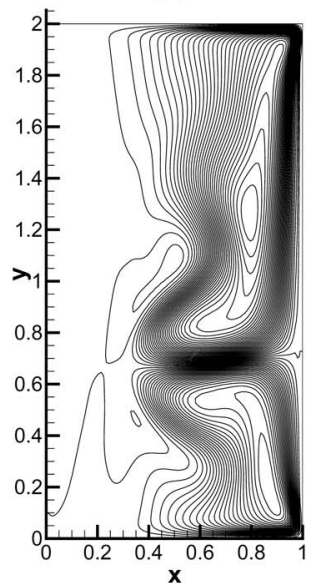

(c)

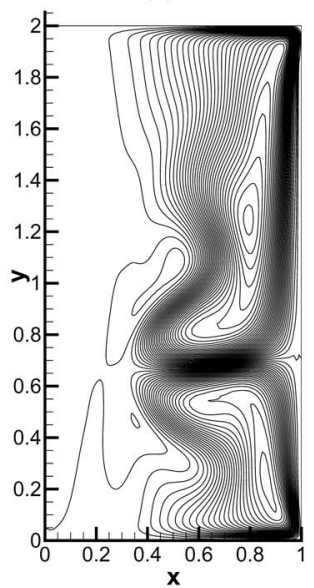

(d)

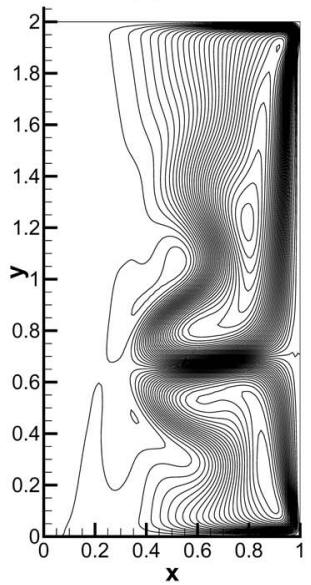

FIG. 16. Streamline plots of steady flow in a closed cylinder with co-rotating bottom at $\xi=0.7$ : (a) $\operatorname{Re}=3500 ;(b) \operatorname{Re}=3800$; (c) $\operatorname{Re}=4000 ;$ and (d) $\operatorname{Re}$ $=4300$.

Reynolds number but the width of the $\mathrm{S}$ shape in co-rotation branch is overpredicted. This transformation of the $\mathrm{S}$ shape is caused by the change in aspect ratio from 1.5 to 2 and therefore the corresponding topological behavior of the transition is different.
The bifurcation from a steady to an unsteady regime is governed by Hopf bifurcations in most of the counter and co-rotating regions. In a region close to the top of the $\mathrm{S}$ shape, however, a discontinuous bifurcation has been detected. (a)

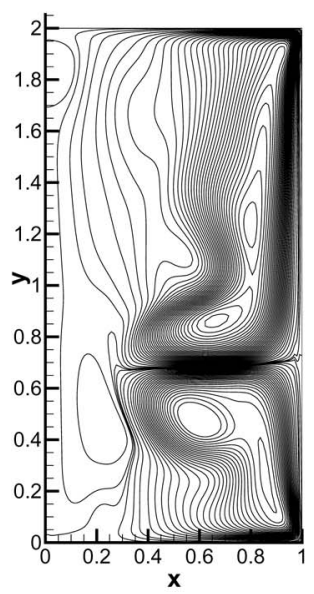

(e)

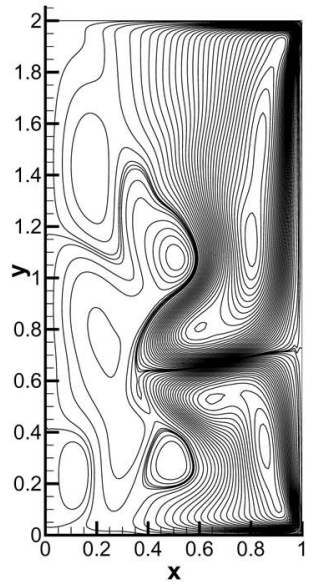

(b)

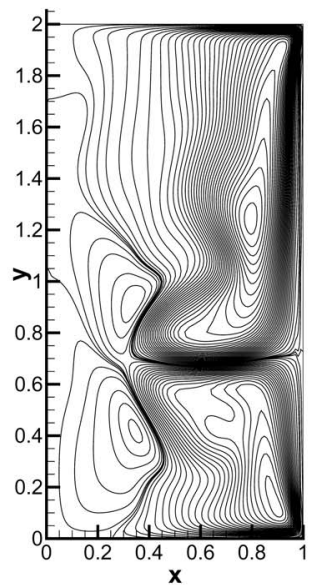

(f)

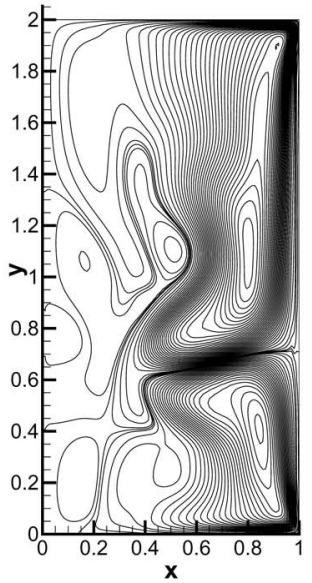

(c)

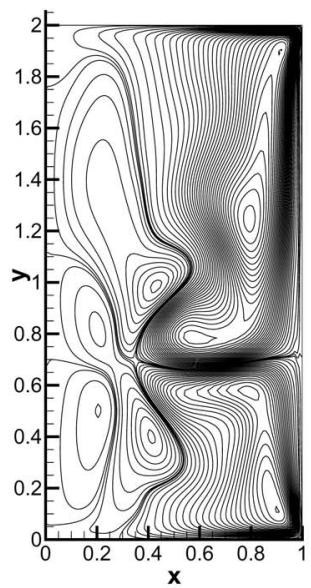

(g)

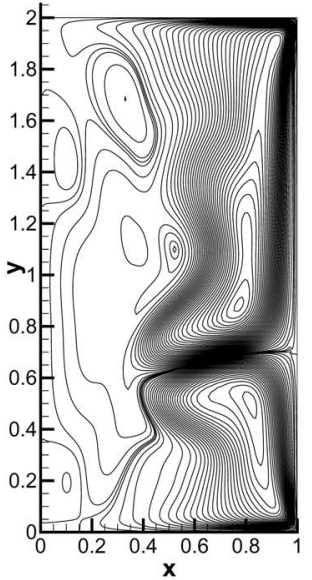

(d)

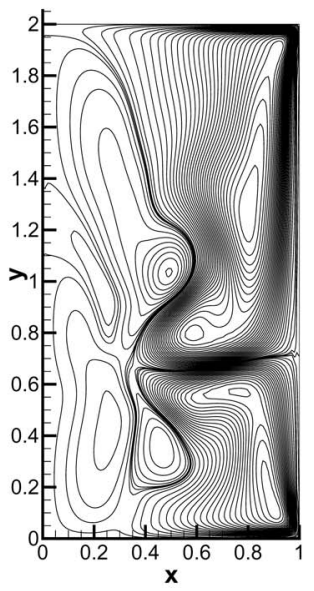

(h)

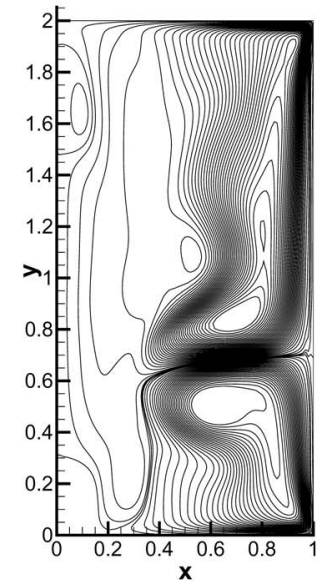

FIG. 17. Stream line plots for unsteady swirl flow in a closed cylinder with co-rotating bottom at $\operatorname{Re}=4400, \xi=0.7$. (a) $t=t_{0}$; (b) $t=t_{0}+T / 8$; (c) $t=t_{0}+T / 4$; (d) $t=t_{0}+3 T / 8$; (e) $t=t_{0}+T / 2$; (f) $t=t_{0}+5 T / 8$; (g) $t=t_{0}+3 T / 4$; and (h) $t=t_{0}+7 T / 8$. 
${ }^{1}$ H. U. Vogel, "Experimentelle Ergebnisse über die laminare Strömung in einem zylindrischen Gehäuse mit darin rotierender scheibe," Max-PlanckInstitut für Strömungsforschung, Göttingen, Bericht 6, 1968 (in German). ${ }^{2}$ B. Ronnenberg, "Ein selbstjustierendes 3-Komponenten-Laserdoppleranemometer nach dem Vergeichsstrahlvefahren, angewandt auf Untersuchungen in einer stationären zylinder-symmetrischen Drehströmung mit einen Rückströmgebiet," Max-Planck-Institut für Strömungsforschung, Göttingen, Bericht 19, 1977 (in German).

${ }^{3}$ M. P. Escudier, "Observations of the flow produced in cylindrical container by a rotating endwall," Exp. Fluids 2, 189 (1984).

${ }^{4}$ A. Spohn, M. Mory, and E. J. Hopfinger, "Experiments on vortex breakdown in a confined flow generated by a rotating disk," J. Fluid Mech. 370, 73 (1998).

${ }^{5}$ F. Sotiropoulos, D. R. Webster, and T. C. Lackey, "Experiments on Lagrangian transport in steady vortex-breakdown bubbles in a confined swirling flow," J. Fluid Mech. 466, 215 (2002).

${ }^{6}$ J. M. Lopez, "Axisymmetric vortex breakdown. Part 1. Confined swirling flow," J. Fluid Mech. 221, 533 (1990).

${ }^{7}$ G. L. Brown and J. M. Lopez, "Axisymmetric vortex breakdown. Part 2. Physical mechanisms," J. Fluid Mech. 221, 553 (1990).

${ }^{8}$ O. Daube, "Numerical simulation of axisymmetric vortex breakdown in a closed cylinder," Lect. Appl. Math. 308, 131 (1991).

${ }^{9}$ J. M. Lopez and A. D. Perry, "Axisymmetric vortex breakdown, Part 3: Onset of periodic flow and chaotic advection," J. Fluid Mech. 234, 449 (1992).

${ }^{10}$ J. N. Sørensen and E. A. Christensen, "Direct numerical simulation of rotating fluid flow in a closed cylinder," Phys. Fluids 7, 764 (1995).

${ }^{11}$ J. P. Watson and G. P. Neitzel, "Numerical evaluation of a vortexbreakdown criterion," Phys. Fluids 8, 3063 (1996).

${ }^{12}$ A. Y. Gelfgat, P. Z. Bar-Yoseph, and A. Solan, "Three-dimensional instability of axi-symmetric flow in rotating lid-cylinder enclosure," J. Fluid Mech. 438, 363 (2001).

${ }^{13} \mathrm{~N}$. Tsitverblit and E. Kit, "On the onset of unsteadiness in confined vortex flows," Fluid Dyn. Res. 23, 125 (1998).

${ }^{14}$ F. Sotiropoulos and Y. Ventikos, "The three-dimensional structure of confined swirling flows with vortex breakdown," J. Fluid Mech. 426, 155 (2001).
${ }^{15}$ F. Sotiropoulos, Y. Ventikos, and T. C. Lackey, "Chaotic advection in three-dimensional stationary vortex-breakdown bubbles: Šil'nikov's chaos and the devil's staircase," J. Fluid Mech. 444, 257 (2001).

${ }^{16}$ H. M. Blackburn and J. M. Lopez, "Modulated rotating waves in an enclosed swirling flow," J. Fluid Mech. 465, 33 (2002).

${ }^{17}$ E. Serre and P. Bontoux, "Vortex breakdown in a three-dimensional swirling flow," J. Fluid Mech. 459, 347 (2002).

${ }^{18}$ H. M. Blackburn, "Three-dimensional instability and state selection in an oscillatory axisymmetric swirling flow," Phys. Fluids 14, 3983 (2002).

${ }^{19}$ P. Z. Bar-Yoseph, A. Solan, and K. G. Roesner, "Recirculation zones in a cylinder with rotating lid," Z. Angew. Math. Mech. 70, 442 (1990).

${ }^{20}$ G. Gautier, P. Gondret, F. Moisy, and M. Rabaud, "Instabilities in the flow between co- and counter-rotating disks," J. Fluid Mech. 473, 1 (2002).

${ }^{21}$ K. Fujimura, H. S. Koyama, and J. M. Hyun, "An experimental study on vortex breakdown in a differentially-rotating cylindrical container," Exp. Fluids 36, 399 (2004)

${ }^{22}$ D. T. Valentine and C. C. Jahnke, "Flows induced in a cylinder with both walls rotating," Phys. Fluids 6, 2702 (1994).

${ }^{23} \mathrm{~J}$. M. Lopez, "Unsteady swirling flow in an enclosed cylinder with reflection symmetry," Phys. Fluids 7, 2700 (1995).

${ }^{24}$ C. Nore, L. S. Tuckerman, O. Daube, and S. Xin, "The 1:2 mode interaction in exactly counter-rotating von Karman swirling flow," J. Fluid Mech. 477, 51 (2003).

${ }^{25}$ A. Y. Gelfgat, P. Z. Bar-Yoseph, and A. Solan, "Steady state and oscillatory instability of swirling flow in a cylinder with rotating top and bottom," Phys. Fluids 8, 2614 (1996).

${ }^{26}$ J. A. Michelsen, "Basis3D-a platform for development of multi-block PDE solvers," AFM Report No. 92-05, Technical University of Denmark, Lyngby, Denmark, 1992.

${ }^{27}$ N. N. Sørensen, "General purpose flow solver applied over hills," RIS $\varnothing$ R-827-(EN), Ris $\varnothing$ National Laboratory, Roskilde, Denmark, 1995.

${ }^{28}$ W. Z. Shen, J. A. Michelsen, and J. N. Sørensen, "Improved Rhie-Chow interpolation for unsteady flow computations," AIAA J. 39, 2406 (2001).

${ }^{29}$ W. Z. Shen, J. A. Michelsen, N. N. Sørensen, and J. N. Sørensen, "An improved SIMPLEC method on collocated grids for steady and unsteady flow computations," Numer. Heat Transfer, Part B 43, 221 (2003). 